Review Article

\title{
The Immunomodulatory Effect of the Gut Microbiota in Kidney Disease
}

\author{
Mingxuan Chi, ${ }^{1}$ Kuai Ma, ${ }^{2}$ Jing Wang, ${ }^{3}$ Zhaolun Ding, ${ }^{4}$ Yunlong Li, ${ }^{1}$ Shaomi Zhu, ${ }^{1}$ \\ Xin Liang, ${ }^{1}$ Qinxiu Zhang $₫$, ${ }^{1}$ Linjiang Song $\odot{ }^{1}{ }^{1}$ and Chi Liu ${ }^{1,5,6}$ \\ ${ }^{1}$ Reproductive \& Women-Children Hospital, School of Medical and Life Sciences, Chengdu University of Traditional \\ Chinese Medicine, Chengdu, China \\ ${ }^{2}$ Department of Nephrology, Osaka University Graduate School of Medicine, Osaka, Japan \\ ${ }^{3}$ Reproductive Center, Shuguang Hospital Affiliated to Shanghai University of Traditional Chinese Medicine, Shanghai, China \\ ${ }^{4}$ Department of Emergency Surgery, Shaanxi Provincial People's Hospital, Xi'an, Shaanxi, China \\ ${ }^{5}$ Department of Nephrology, Sichuan Clinical Research Center for Kidney Disease, Sichuan Provincial People's Hospital, University of \\ Electronic Science and Technology, Chengdu, China \\ ${ }^{6}$ Chinese Academy of Sciences Sichuan Translational Medicine Research Hospital, Chengdu, China
}

Correspondence should be addressed to Linjiang Song; linjsong_scu@163.com and Chi Liu; liuchi1985@163.com

Mingxuan Chi and Kuai Ma contributed equally to this work.

Received 12 February 2021; Revised 26 March 2021; Accepted 20 April 2021; Published 15 May 2021

Academic Editor: Ruirong Tan

Copyright ( 2021 Mingxuan Chi et al. This is an open access article distributed under the Creative Commons Attribution License, which permits unrestricted use, distribution, and reproduction in any medium, provided the original work is properly cited.

\begin{abstract}
The human gut microbiota is a complex cluster composed of 100 trillion microorganisms, which holds a symbiotic relationship with the host under normal circumstances. Intestinal flora can facilitate the treatment of human metabolic dysfunctions and interact with the intestinal tract, which could influence intestinal tolerance, immunity, and sensitivity to inflammation. In recent years, significant interests have evolved on the association of intestinal microbiota and kidney diseases within the academic circle. Abnormal changes in intestinal microbiota, known as dysbiosis, can affect the integrity of the intestinal barrier, resulting in the bacterial translocation, production, and accumulation of dysbiotic gut-derived metabolites, such as urea, indoxyl sulfate (IS), and p-cresyl sulfate (PCS). These processes lead to the abnormal activation of immune cells; overproduction of antibodies, immune complexes, and inflammatory factors; and inflammatory cell infiltration that can directly or indirectly cause damage to the renal parenchyma. The aim of this review is to summarize the role of intestinal flora in the development and progression of several renal diseases, such as lupus nephritis, chronic kidney disease, diabetic nephropathy, and renal ischemia-reperfusion injury. Further research on these mechanisms should provide insights into the therapeutic potential of regulating intestinal flora and intervening related molecular targets for the abovementioned nephropathy.
\end{abstract}

\section{Introduction}

Kidney disease is a general term for renal heterogeneous disorders affecting the kidney structure and function, which is a dominant contributor to global morbidity and mortality [1]. Although it has been increasingly identified as a significant public health problem worldwide with increasing prevalence and poor outcomes, clinical diagnosis and therapeutic interventions are lagging. Nowadays, most therapeutic methods are limited to lowering blood pressure, controlling blood glu- cose, and reducing proteinuria [2]. Current studies are aimed at developing more effective therapeutic strategies to prevent the progression of renal diseases. Over the past years, our understanding of the composition and function of the gut microbiota has been expanded, mainly on account of the evolution and advances of modern molecular techniques. Developing research studies on gut microbiota have shown that this formerly "neglected organ" plays a significant role in many diseases within and beyond the intestinal tract. Substantial differences of the gut microbiota composition, immunogenicity, 
and metabolic activity have been observed by comparing healthy individuals to patients presenting with different types of kidney diseases [3-6] and other noncommunicable illnesses, such as diabetes mellitus, obesity, atherosclerotic cardiovascular disease, heart failure, and liver diseases [7-11]. These changes of the gut microbiota are causally correlated with disease phenotypes, complications, and outcomes according to experimental studies in animals and humans [7, 12, 13].

The human gut microbiota, also known as the intestinal flora, is composed of $\sim 100$ trillion microorganisms constituted by a broad spectrum of over 500 genera of bacteria from two main phyla, namely, Bacteroidetes and Firmicutes. Generally, the diversity and abundance of the intestinal microbiota differ along the intestinal tract and maintain a dynamic balance. Known as the body's "second brain," the intestinal microbiota plays a major role in the absorption and metabolism of nutrients, hormone secretion, and toxin degradation, which enable it to control the human intestinal homeostasis and even the whole internal environment. The SCFAs including acetate, propionate, and butyrate are predominant final products of the distal gut microbiome, fermented from a variety of plant polysaccharides produced under anaerobic conditions [14]. SCFAs can provide about $10 \%$ of the caloric needs for the human body [15] and enhance the barrier function by regulating the retinol production and mediating the secretion of mucin and IgA [16]. In addition, SCFAs come into the blood circulation and then exert their systemic effects such as increasing anti-inflammatory factors, downregulating autoimmunityrelated factors, and developing regulatory $\mathrm{T}$ (Treg) cells [1720] via the $G$ protein-coupled receptors (e.g., GPR41, GPR43, and GPR109A). Furthermore, accumulating pieces of evidence have reported the positive effects of SCFAs in treating kidney problems caused by several diseases [13, 21-24].

In addition to the metabolic function, the gut microbiota performs some basic roles to promote the maturation of intestinal immunity [25-28] and maintain the integrity of the intestinal epithelial barrier to prevent the invasion and colonization of pathogenic microorganisms [29]. The intestinal epithelial barrier is essential for intestinal homeostasis, which enables the bilateral passage of vast metabolites and immune signals and simultaneously obstructs the passage of the pathogenic bacteria, toxic metabolites, and microbial byproducts [30-32]. To counterpoise these apparently contradictory roles $[33,34]$, the epithelial cells and immune cells closely interact with each other, establishing the first line of protection against invading pathogens. Its mechanism of action is the recruitment of phagocytes or direct bacterial prevention and killing by releasing chemokines, cytokines, AMPs, and other soluble molecules [35-38]. The intestinal immune system is built up and matures with the participation of the gut microbiota. Microfold cells ( $M$ cells) in the epithelium capture lumen contents and deliver them to the underlying antigen-presenting cells, such as macrophages and dendritic cells (DCs) [39]. Once the pattern recognition receptors of the DCs bind to the pathogenic microorganisms, the stimulated DCs that process and present the antigens express costimulatory molecules and cellular factors. These processes contribute to the regulating helper $\mathrm{T}$ (Th) cells, such as Th1, Th2, and Th17, and immunosuppressive Treg cells differentiated from naive $\mathrm{CD}^{+} \mathrm{T}$ cells, maintaining the Treg/Th17 balance and the immune homeostasis [14, 40-42] (Figure 1). Intestinal flora imbalance induces the activation of immune cells through this pathway, secreting a large amount of proinflammatory factors (e.g., IL-4, IL-5, IL-6, and interferon- $\gamma(\operatorname{IFN}-\gamma))$, which results in immune dysregulation and inflammation. The intestinal microbiome promotes the differentiation of IgA-secreting plasma cells by activating a proliferation-inducing ligand (APRIL) receptor and B cell-activating factor (BAFF) in DCs. sIgA has a regulatory effect on intestinal microorganisms. Bacterial metabolites, such as SCFAs, histamine, spermine, and taurine, can also influence the host's immune homeostasis [43].

As a contributing factor and indicator of human health, the gut microbiota plays an important role in the prevention, diagnosis, and treatment of many human diseases. Although a dynamic balance is established between intestinal flora, host, and external environment, it is susceptible to changes caused by age, diet, antibacterial drugs, psychological pressure, and other factors, resulting in an imbalance of intestinal flora [44, 45]. Once this microecological balance of intestinal flora is destroyed, known as dysbiosis, it will lead to a variety of gastrointestinal and systemic diseases (Figure 1). Microbial dysbiosis promotes the production of bacteria-produced uremic toxins, such as IS, p-cresyl sulfate (PCS), and trimethylamine N-oxide (TMAO). These metabolites translocate into the circulation through the impaired intestinal barrier, and most of which are excreted by the kidneys, where their retention would lead to kidney dysfunction [46]. In addition to dysbiosis, the increased permeability and structural damage of the intestinal barrier result in the translocation of pathogenic bacteria and their byproducts which is a vital step leading to local or systemic inflammation [47-50], affecting various organs, including the kidneys [51, 52].

Taken together, the colonization of intestinal microorganisms is a double-edged sword for the host. The healthy microbial community plays an indispensable role in the host's nutrient absorption and metabolism, the maturation of intestinal immunity, the maintenance of the integrity of the intestinal epithelial barrier, and the prevention of colonization by pathogenic microorganisms. These are what the microbiome has contributed to the overall health of the host. However, changes in the intestinal flora can cause diseases of different organs and exacerbate existing diseases. This review summarizes the current understanding of the role of intestinal flora in the occurrence and development of kidney disease, focusing on select components of the immune system that have been shown to drive the pathogenesis of each kidney disease. Further research on the association between the immune system and the gut microbiota may contribute to the understanding of the intricate pathogenesis of kidney disease. Likewise, the regulation of intestinal flora and the intervention of related molecular targets may have a potential therapeutic utility in the treatment of kidney diseases.

\section{Gut Microbiota in Lupus Nephritis}

Systemic lupus erythematosus (SLE) is a multisystemic autoimmune disease characterized by lymphocyte overactivation and the production of antinuclear autoantibodies that drive 


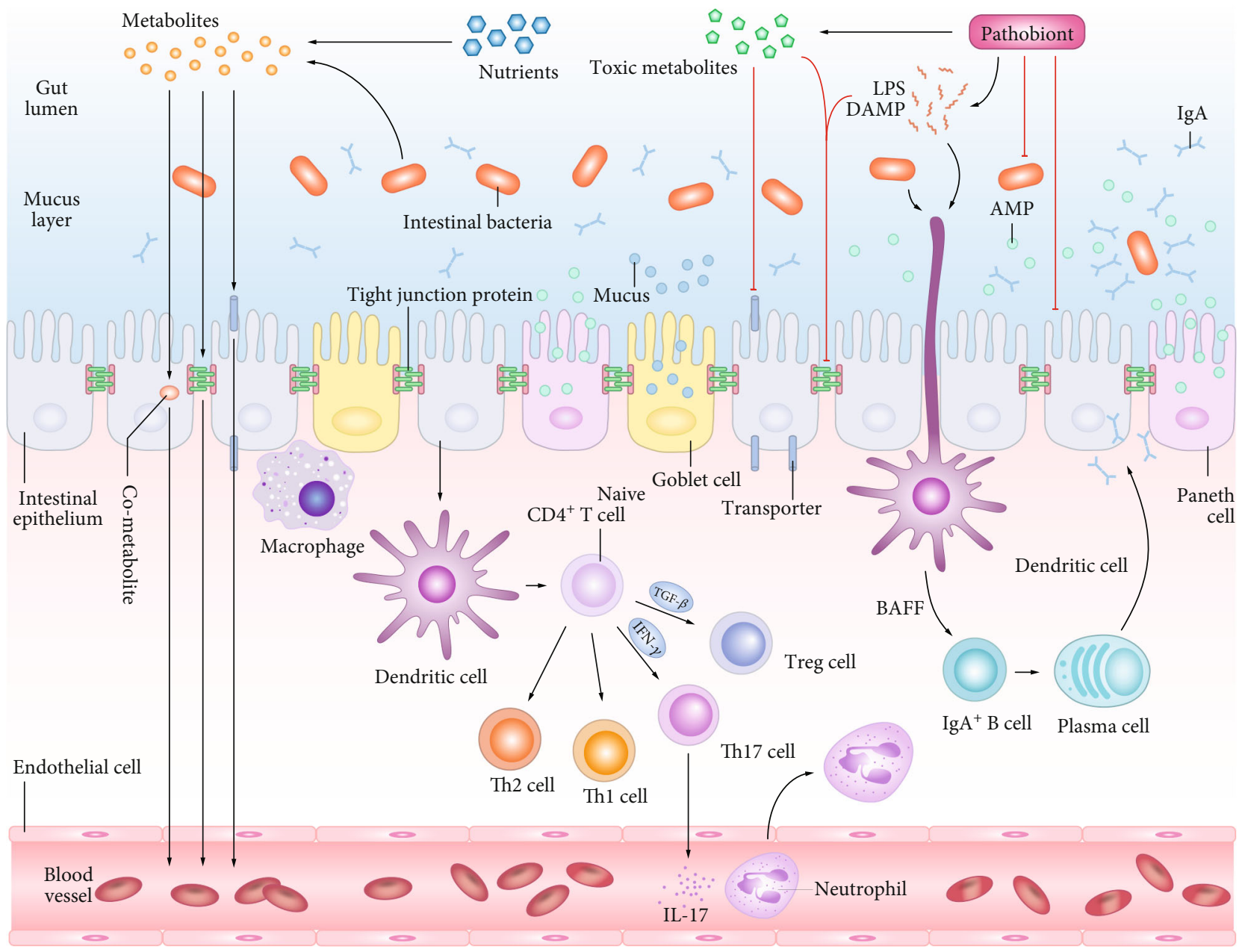

FIGURE 1: The intestinal epithelial barrier allows a large number of metabolites and immune signals to pass in both directions while blocking the pathways of pathogenic bacteria, toxic metabolites, and microbial byproducts. The outermost layer of the intestinal barrier is the mucus layer which is composed of mucin glycoprotein, AMPs, and sIgA, produced by goblet cells, Paneth cells, and plasma cells, respectively, and excludes the microbiome from the epithelial surface. Adjacent cells are linked together by the tight junction protein families that can determine the permeability and prevent mechanical disruption of the epithelial sheet. The intestinal immune system is established and mature with the participation of the gut microbiota. Once bound to luminal antigens, DC pattern recognition receptors express costimulatory molecules and cellular factors involved in regulating Th1, Th2, Th17, and Treg cells differentiated from naive CD4 ${ }^{+} \mathrm{T}$ cells, maintaining Treg/Th17 balance and forming immune homeostasis. The intestinal microbiome promotes the differentiation of IgAsecreting plasma cells by activating APRIL (a proliferation-inducing ligand) receptor and B cell-activating factor in DC.

arthritis, glomerulonephritis, and other different inflammatory tissue damage [53]. Approximately $60 \%$ of SLE patients are suffering from lupus nephritis (LN), which is one of the leading causes of morbidity and mortality in SLE, resulting in acute or chronic kidney damage through inflammation, deposition of immune complexes, and glomerular or interstitial scarring [54]. Nowadays, the etiological understanding of $\mathrm{LN}$ is limited in the genes and environment [55], but the specific causes still remain unclear. In recent years, the alterations of the gut microbiota have been associated with multitudinous autoimmune disorders, and present data has reported the distinctive microbiota composition in the gastrointestinal tract of LN patients [3,56-61]. Thus, the role of intestinal flora in LN has increasingly attracted the attention of researchers [62].

In the symbiotic condition, intestinal microorganisms can affect gut tolerance, immunity, and sensitivity to inflam- mation through B cell maturation, Treg/Th17 ratio balance, and anti-inflammatory cytokine secretion. However, the intestinal inflammatory microenvironment in SLE patients may influence intestinal tolerance, exceeding the immunologic reactions, autoimmunity, and damage of tissues/organs in SLE patients. In the pathological circumstances of SLE, chronic inflammation disrupts the intestinal barrier, which is termed as leaky gut [63], and bacterial pathogens are directly exposed to various organs and immune systems of the body. Through the toll-like receptor (TLRs) [64, 65], antigen-presenting cells (APCs, e.g., macrophages and DCs) secrete cytokines to activate the differentiation and proliferation of T cells $[14,40]$. Proinflammatory factors, such as IL-6 and IFN- $\alpha$, are released, which play an important role in inducing $\mathrm{B}$ cells to release autoantibodies and cause an imbalance in the Treg/Th17 ratio [66]. A large number of autoantibodies, most of which are anticellular antibodies 


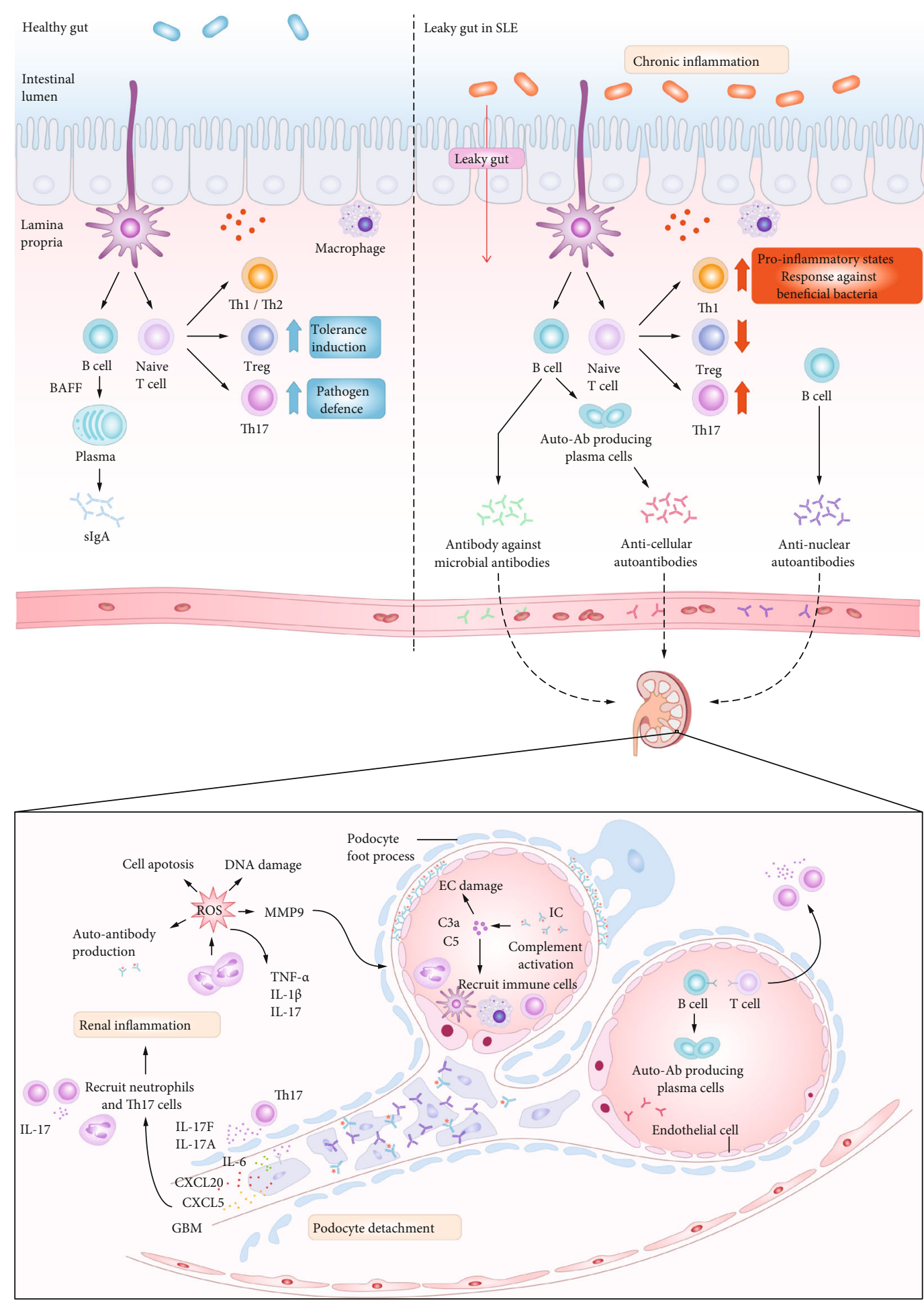

FIGURE 2: Correlation between intestinal microbiota and the incidence of lupus nephritis. Under the symbiotic condition, intestinal microorganisms can affect gut tolerance, immunity, and sensitivity to inflammation through B cell maturation, Treg/Th17 ratio balance, and anti-inflammatory cytokine secretion. In SLE patients, the intestinal inflammatory atmosphere can induce B cells to release autoantibodies, resulting in an imbalance of the Treg/Th17 ratio, leading to intestinal tolerance disorders, beyond immune response and autoimmunity, and tissue/organ damage (such as lupus arthritis (LN)). A large number of autoantibodies and immune complexes are produced and enter the circulation. The deposition of autoantibodies and immune complexes in the glomeruli leads to the activation of complement components (e.g., C3a and C5), resulting in the endothelial cell or podocyte injury and recruitment of immune cells. Infiltrated Th17 cells in the kidney secrete cytokines IL-17A and IL-17F, which activate mesangial cells and tubular epithelial cells to produce CXCL5 and CCL20, then recruit more Th17 cells and neutrophils through CCR6 and CXCR2, respectively. At the same time, ROS produced by infiltration of immune cells can lead to further renal inflammation and tissue destruction. 
and antinuclear antibodies [67-69], combine with ligands to form immune complexes. Moreover, molecular mimicry may be an important link between intestinal microbiota and SLE. Bacteria can express orthologs of human Ro60 autoantigens in the SLE patients' gut. This characteristic would lead to T cell cross-reaction and the production of human anti-Ro60 autoantibodies in SLE patients [70, 71]. Particularly, it was found that Ruminococcus gnavus cross-reacts with human DNA, whose relative abundance in the intestinal tract is positively correlated with SLE activity and LN [72].

These changes initiated by the gut microbiota can lead to an acceleration of the process of kidney injury. The deposition of autoantibodies and immune complexes in the glomeruli leads to the activation of complement components, resulting in the injury of endothelial cells or podocytes and the recruitment of immune cells. Some pieces of evidence have reported the essential role of renal resident cells (e.g., podocytes, renal tubular epithelial cells, and glomerular mesangial cells) in the development of LN [73]. Infiltrated Th17 cells in the kidney secrete cytokines IL-17A and IL$17 \mathrm{~F}$, which activate the mesangial cells and tubular epithelial cells to produce C-X-C motif chemokine 5 (CXCL5) and chemokine (C-C motif) ligand 20 (CCL20) and then recruit more Th17 and neutrophils through chemokine receptor 6 (CCR6) and CXCR2, respectively. At the same time, reactive oxygen species (ROS) produced by the infiltration of immune cells can lead to further renal inflammation and tissue destruction (Figure 2).

The current treatment for LN includes the administration of high doses of corticosteroids and broad-spectrum immunosuppressants, but treatments are not ideal at present [74]. The study of the interaction between intestinal microflora and LN provides a new idea for the treatment of LN. In experimental studies, the restoration of the composition of intestinal flora through the administration of acidic water [67], vitamin A [61], probiotics [3, 75, 76], or prebiotics can moderate the inflammatory status and possibly favor renal protection in SLE models. Rodgers et al. demonstrated the renal protective potential of drug-like analogs of ES-62, which is a type of phosphorylated cholinergic glycoprotein secreted by Acanthocheilonema viteae and involved in maintaining the balance of regulatory/effector B cells and desensitized renal effector function [77]. Since limited reports have investigated the impact of the abovementioned treatments in SLE and LN patients, further research is needed to confirm their efficacy in clinical application.

\section{Gut Microbiota in Chronic Kidney Disease}

Chronic kidney disease (CKD) is a global health issue and is increasingly considered a social burden. More than $10 \%$ of the population has been diagnosed with CKD, in which $50 \%$ are classified as high-risk subgroups [78]. As a result of progressive renal parenchymal injury, clinical symptoms, such as the reduced glomerular filtration rate, increased urinary protein excretion, reduced synthesis of erythropoietin, and hypertension, can be noticed in patients with CKD.

CKD induces numerous alterations in internal and external factors that potentially alter the microbiota composition.
Furthermore, intestinal dysbiosis is closely associated with gut inflammation and intestinal barrier disruption [79, 80]. For instance, dietary changes in CKD patients might contribute to intestinal dysbiosis and the generation of excessive uremic toxins. Urea is produced from amino acids in the urea cycle and is excreted by the kidneys $(80 \%)$ and the digestive tract $(20 \%)$. As renal function is impaired in patients with $\mathrm{CKD}$, the digestive tract becomes the main route for urea excretion. Urea in the intestinal lumen could be converted by bacteria to $\mathrm{NH}_{3}$ or $\mathrm{NH}_{4} \mathrm{OH}$, wherein the formation of which and increased intestinal lumen $\mathrm{pH}$ can promote the proliferation of pathogenic microorganisms and destroy the intestinal barrier. Other causes that probably contribute to intestinal barrier disruption in CKD include the use of numerous medications and hypervolemia $[81,82]$ which lead to uremia, azotemia sympathetic overactivity $[83,84]$, and intestinal congestion [5]. These processes lead to systemic inflammatory responses through increasing the production of proinflammatory cytokines, activating the nuclear factorkappa $\mathrm{B}(\mathrm{NF}-\kappa \mathrm{B})$ pathway, and dysregulating the immune response, thus exacerbating the ecological imbalance [79, 85]. The destruction of the intestinal barrier facilitates the bacterial endotoxin to enter the circulatory system, which is known as endotoxin translocation. Endotoxemia has various effects on systemic inflammation, oxidative stress, cardiac injury, and atherosclerosis [52]. More importantly, endotoxemia is positively correlated with the reduced survival of CKD and hemodialysis patients [52]. The inflammation in CKD involves the endotoxin-induced overactivation of APCs and lymphocytes [86]. However, evidence suggested that host defense against infectious microorganisms is impaired in end-stage renal disease (ESRD) patients [87, 88]. This seemingly paradoxical immune response can be explained by the endotoxin tolerance; that is, persistent innate immune activation induces immune paralysis [89], which contributes to the presence of acquired immunosuppression and systemic inflammation.

In the liver and colon, dysbiotic gut-derived uremic toxins, such as indoles and phenols, are further metabolized into TMAO, IS, and PCS $[80,90]$. They enter the circulation through the impaired intestinal barrier and then exert harmful effects on the kidney. IS has the ability to promote the production of ROS in renal tubular epithelial cells; activate NF$\kappa \mathrm{B}, \mathrm{p} 53$, and other regulatory factors; and upregulate the expression of chemokines, leading to the aggregation of renal interstitial monocytes/macrophages and finally causing renal fibrosis [91]. IS can also promote the expression of transforming growth factor- $\beta$ (TGF- $\beta$ ) and accelerate renal function deterioration by activating the renin-angiotensinaldosterone system (RAAS) [92]. PCS has a proinflammatory effect, which can promote renal interstitial monocyte/macrophage infiltration and upregulate the expression of inflammatory factors, such as IL- 6 and TGF- $\beta$, thus promoting renal fibrosis [93]. Both IS and PCS can lead to the hypermethylation of the Klotho gene, inhibit Klotho gene expression, weaken the protective effect of its products on the kidneys, and ultimately accelerate renal function deterioration [94]. In the circulation, $100 \%$ of the PCS and IS are bound to proteins, which limits their clearance; they could 


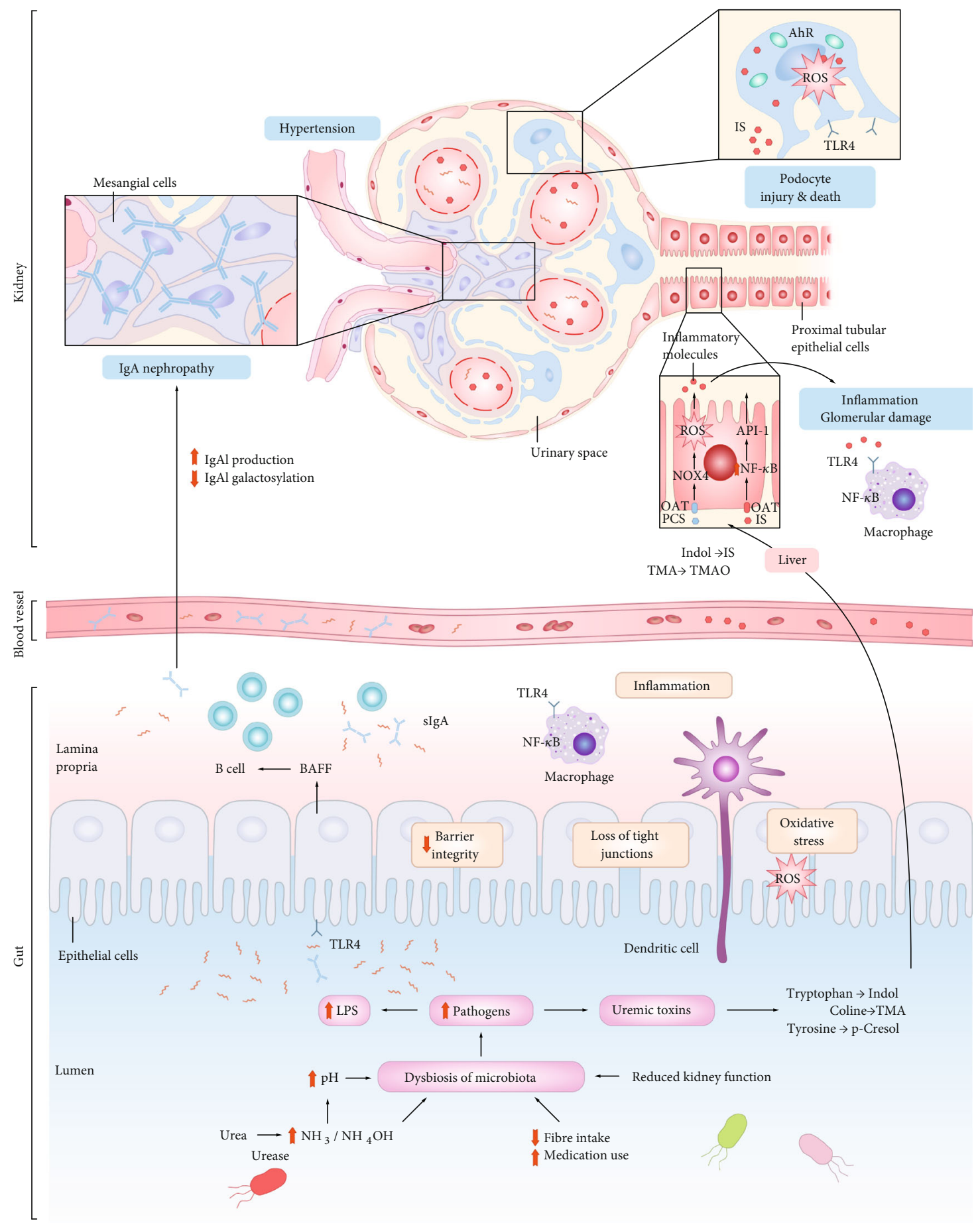

FIGURE 3: Changes in diet, numerous medication use, and decreased kidney function in CKD patients may lead to intestinal dysbiosis. The digestive tract becomes the main route for urea excretion in CKD patients with impaired renal function. A large amount of NH3 or $\mathrm{NH} 4 \mathrm{OH}$ which are produced by bacteria can increase the intestinal $\mathrm{pH}$ value, promote the intestinal dysbiosis and destroy intestinal barrier. Thus, the transport of endotoxins (e.g., lipopolysaccharides (LPSs)) into the bloodstream has different effects on systemic inflammation, oxidative stress, cardiac injury, and atherosclerosis. Dysbiotic gut-derived uremic toxins, such as indoles and phenols, are further metabolized into trimethylamine N-oxide (TMAO), indoxyl sulfate (IS), and p-cresyl sulfate (PCS) in the liver and colon. The introduction of uremic toxins into the circulation causes inflammation and tubulointerstitial damage and promotes ROS production, tubulointerstitial damage, and nephrotoxicity of proximal tubuloepithelial cells. Intestinal microbial metabolites SCFAs are associated with hypertension and are important risk factors for CKD. SCFAs trigger hypertension through Olfr78, leading to renin secretion and regulation of peripheral resistance. In addition, bacteria and their components are involved in the hyperproduction and hypogalactosylation of IgA. 
not be eliminated by dialysis. However, the binding capacity of proteins as a whole is decreased in patients with CKD [95], augmenting the circulating levels of unbound metabolites. The increased levels of PCS and IS in serum were positively correlated with renal degeneration, nephropathy progression, cardiovascular diseases, and mortality in patients with CKD [51]. Collectively, uremic toxins will trigger inflammation and tubulointerstitial damage and promote ROS production, tubular injury, and renal toxicity in the proximal renal tubular epithelial cells [96]. In addition to the tubules and mesenchymal damage, IS also injures the glomeruli where the podocytes play an important role. Podocytes are highly differentiated cells that are involved in the formation of glomerular filtration membranes and have a limited regenerative capacity [22]. Once the podocytes are damaged, proteinuria and other clinical manifestations of renal disease would occur. The abnormal increase of IS induces AhR activation, which contributes to the progressive impairment of the podocytes and glomeruli [97].

Renal disease is inextricably linked to cardiovascular diseases. Overall, $85 \%-90 \%$ of patients with CKD have hypertension [98], which is an important risk factor for CKD. Emerging studies have demonstrated a strong link between gut microbiota and hypertension in animals and patients [84, 99-103]. Yang et al. compared the fecal microbiome of spontaneously hypertensive rats and angiotensin-induced hypertensive rats. They noticed a prominent dysbiosis characterized by decreasing microbial abundance, variety, and evenness and the increased Firmicutes/Bacteroidetes ratio in hypertensive rats [103]. Moreover, treatments with antibiotics can lower the blood pressure of patients with treatment-resistant hypertension [102], indicating that the intestinal microbiome plays a role in hypertension pathogenesis and one of the possible causes is increased gut permeability and translocation of bacterial products [84]. Accumulating pieces of evidence suggest that gut-derived SCFAs contribute to the regulation of blood pressure via olfactory receptor 78 (Olfr78) and GPR41 [23, 84, 101, 104, 105]. Specifically, SCFAs trigger hypertension through Olfr78 in the peripheral blood vessels and renal afferent arterioles, leading to the secretion of renin and modulation of peripheral resistance when an intestinal microbial imbalance occurs. By contrast, SCFAs are able to lower blood pressure by binding with GPR41 and GPR43. Moreover, further research on the relationship between the intestinal microflora and the renal-cardiovascular system is helpful in the development of effective treatment methods for hypertension and CKD (Figure 3).

In view of the role of hypertension and other pathogenesis of CKD, the most useful management in the early stage of CKD is the control of blood pressure, along with reducing protein and salt intake to prevent acute renal injury and control blood glucose levels. With the exception of dialysis and kidney transplantation, no effective strategy to cure or prevent ESRD is currently available. Considering this, it could be hypothesized that regulating the intestinal microbiota can lower blood pressure, ameliorate kidney disease, and prevent complications in patients with CKD. Intervening measures (e.g., increasing fiber intake, rational use of antibiotics
$[106,107]$, and therapeutic use of probiotics, prebiotics, and synbiotics) can restore the composition of intestinal flora and inhibit the accumulation of urotoxins in the blood $[108,109]$. According to the research of Lakshmanan et al. [110], prebiotic gum acacia (GA) treatment restored the intestinal balance of CKD rats and relieves the inflammation of kidney tissue by increased production of butyrate, as well as its anti-inflammatory and antioxidant capacity. Future studies are needed to improve dialysis techniques to isolate protein-bound uremic toxins and to discuss the feasibility of fecal microflora transplantation.

\section{Gut Microbiota in Diabetic Nephropathy}

The worldwide prevalence of diabetes is rising rapidly, and it is estimated to increase to 578 million in 2030 [111]. Diabetes increases the risk of multiple complications, such as decreased kidney function and cardiovascular disease [112]. Although only $30 \%-40 \%$ of diabetic patients develop diabetic nephropathy (DN), it is a leading cause of ESRD in most developed countries and a key determinant of survival in people with diabetes [113]. Pathologically, the major changes in the kidney are the deposition of the extracellular matrix (ECM), thickening of the glomerular basement membrane, tubular atrophy, and cellular proliferation that results in interstitial fibrosis and glomerulosclerosis [113]. Accumulating pieces of evidence have revealed that increased ROS and low-grade inflammation, due in part to hyperglycemia, are strongly associated with diabetic complications $[114,115]$. These changes lead to kidney damage, such as glomerular hyperfiltration, glomerular hypertension, altered glomerular composition, and hypernephrotrophy. Although the relationship is not clear, most studies believe that dysbiosis is involved in the occurrence and development of diabetes and $\mathrm{DN}$, which may be related to the induction of insulin resistance and long-term chronic inflammation in diabetes.

Studies have investigated intestinal dysbiosis among diabetic patients and nondiabetic individuals $[10,11,116]$, and it was found that intestinal dysbiosis is associated with insulin resistance and lipid metabolic disorders [117]. Intestinal dysbiosis itself and abnormal lipid metabolism in diabetes can decrease the expression of connective proteins, resulting in increased intestinal permeability and bacterial translocation. LPSs translocate into the circulation through the dysfunctional barrier and mediate host inflammatory responses through TLR2- and TLR4-related pathways, which is associated with the occurrence and development of many metabolic diseases. Chronic inflammation may also lead to the apoptosis of islet cells and eventually diabetes [7]. He et al. found that probiotics could delay the occurrence and development of diabetes by improving insulin resistance and stabilizing fasting blood glucose (FBG) levels [118]. The abovementioned studies have shown the correlation between intestinal flora and diabetes and found that restoring the gut microbiota is considered to be an effective strategy in preventing and treating diabetes.

In addition to the effect on insulin resistance, the intestinal microbiota may also be closely related to the occurrence and development of renal disease in diabetes through some 
other ways. Although magnesium lithospermate B is unable to decrease the FBG levels in STZ mice, the study of Zhao et al. showed that it can improve renal function (decreasing $24 \mathrm{~h}$ urinary protein) in diabetic patients by restoring the intestinal microbial composition and regulating the bile acid metabolism [119]. In 2019, one study first discovered a direct association between intestinal flora and DN. Through analyzing the fecal flora composition among diabetic biopsyproven DN patients and healthy controls, the researchers found that the composition of the gut microbiota of DN patients is different from diabetic patients and healthy controls with several strains, such as Escherichia-Shigella [4]. The increased abundance of Escherichia-Shigella could penetrate the intestinal barrier and then exacerbate the intestinal leakage [120], which could contribute to the chronic lowgrade inflammatory status in diabetic patients [121]. The interaction of bacterial LPS with TLR2 and TLR4 has been shown to be involved in the ongoing inflammatory process of DN by activating NF- $\kappa \mathrm{B}$ and inducing the release of proinflammatory cytokines (TNF, IL-1, IL-6, etc.) in an inflammatory cascade that exacerbates renal damage [118]. Moreover, the accumulation of toxic metabolites produced by intestinal microorganisms stimulates the production of ROS through the NADPH pathway, which triggers the NF- $\kappa$ B pathway and induces an inflammatory response, and contributes to proteinuria and podocyte damage. Kikuchi et al. suggested that phenyl sulfate, a type of bacterial toxin, could potentially be an early diagnostic marker and a therapeutic target of DN in the future [122]. The NF- $\kappa$ B pathway is a key point for the progression of inflammation and fibrosis in DN, whose activation can reduce the expression of inflammatory cytokines and fibrosis degree [123].

Recent studies have focused on the relationship between enterogenic products such as SCFAs and DN, which is a hot research field recently. Lu et al. speculated that intestinal microorganisms produce excessive SCFAs, especially acetate, which could bind to the renal Olfr78 receptor, and activate the intrarenal renin-angiotensin system (RAS) [124]. The activation of the RAS has long been regarded as one of the initiators of DN. The kidney is sensitive to angiotensin II (Ang II) which leads to renal vasoconstriction, increased blood pressure, and glomerular hypertension [125]. Moreover, Ang II promotes the morphological changes of podocytes and glomerular endothelial cells, the deposition of the extracellular matrix, and the secretion of inflammatory factors and profibrotic chemokines, accelerating the progress of DN. Furthermore, Hu et al. demonstrated that the acetate produced by the intestinal flora mediates the dysregulation of cholesterol homeostasis by activating GPR43, which leads to the tubulointerstitial injury of DN [126].

Clinical studies have demonstrated that traditional treatments that control glucose levels and inhibit RAS and inflammation could not absolutely prevent the progression of renal damage in DN. Considering this, the gut microbial factors could be involved in the pathogenesis of DN besides the traditional risk factors [127-129]. Modulating the gut microbiota may lead to better glycemic control and favorable outcomes in people with diabetes. Probiotics and prebiotics (e.g., fructooligosaccharides, lactulose, inulin, and resistant starches) are commonly used to regulate the gut flora, and the application of synbiotics and probiotics has been found to regulate the metabolic profile (e.g., glycemic, blood pressure, and lipid profile) of people with diabetes [130]. Studies have also reported the effect of probiotics and synbiotics in decreasing the biomarkers of inflammatory factors and oxidative stress $[24,131,132]$, which could ameliorate kidney injury in diabetes. Chinese herbal medicine, QiDiTangShen granules, has been confirmed to modulate the gut microbiome composition and improved bile acid profiles in a mouse model of DN [133]. These benefits could be attributed to the ability of probiotics to restore epithelial barriers, producing SCFAs, modulating the immune response locally and systemically, and improving the gut barrier function. Moreover, in patients with type 2 diabetes, dietary fiber intake was strongly associated with glycemic control [11] and negatively associated with the prevalence of metabolic syndrome, both of which were associated with a lower risk of renal disease [134].

\section{Gut Microbiota in Renal Ischemia- Reperfusion Injury}

Renal ischemia-reperfusion injury (IRI) contributes to acute kidney injury (AKI) and delayed graft function after kidney transplantation [135]. Blood reperfusion of ischemic tissue increases the production of ROS that could attack the cells and tissue. Endogenous danger signals are released after cell stress and death, which could activate the tubules and endothelial cells to enhance the expression of adhesion molecules that can recruit innate and adaptive immune cells and promote ROS production [136]. Moreover, excessive ROS destroy the ratio of oxidant/antioxidant enzymes, leading to mitochondria-mediated cell apoptosis. Tubular epithelial cells and APCs secrete cytokines and chemokines that lead to the inflammatory response. APCs, such as macrophages and DCs, could activate $\mathrm{CD} 4^{+}$and $\mathrm{CD} 8^{+} \mathrm{T}$ lymphocytes by increasing the expression of total stimulus molecules, thereby leading to tissue damage [137].

According to the study of Emal et al., applying antibiotics leads to the diminution of the gut microbiome that can profoundly protect against kidney IRI by reducing the maturation status of the bone marrow monocytes and $\mathrm{F} 4 / 80^{+}$renal resident macrophages [138], suggesting that intestinal microbes play a role in the progression of ischemiareperfusion injury to AKI. Furthermore, the treatment with the SCFAs (acetate, propionate, and butyrate) that the gut bacteria produced in the distal colon can improve renal dysfunction in mice with IRI. This protection was associated with the functions of SCFAs, such as reducing inflammation, cellular oxidative stress, and immune cell infiltration and regulate DNA methylation status [21].

\section{Gut Microbiota in IgA Nephropathy}

IgA nephropathy (IgAN) is the most common type of glomerulonephritis globally and a dominant cause of CKD and renal failure [139]. A characteristic of IgAN patients is the circulating elevation and glomerular accumulation of 
TABLE 1: The related mechanism in the relationship between gut microbiota and kidney diseases.

\begin{tabular}{|c|c|c|c|}
\hline $\begin{array}{l}\text { Kidney } \\
\text { diseases }\end{array}$ & Related mechanism & Conclusions & References \\
\hline \multirow{5}{*}{$\mathrm{LN}$} & Molecular mimicry & $\begin{array}{l}\text { In susceptible individuals, symbiotic bacterial antigens cross-react with } \\
\text { human DNA to activate the immune system and destroy self-tolerance, } \\
\text { which is positively correlated with SLE activity and LN. }\end{array}$ & {$[70,71]$} \\
\hline & Treg/Th17 imbalance & $\begin{array}{c}\text { Treg/Th17 imbalance can trigger immune responses and promote the } \\
\text { production of SLE autoantibodies. }\end{array}$ & {$[40,42]$} \\
\hline & 个TLR7 and TLR9 & $\begin{array}{c}\text { An increase of TLR7 and TLR9 can contribute to alterations of } \\
\text { proinflammatory cytokines in lupus patients. }\end{array}$ & {$[64,65]$} \\
\hline & \multirow{2}{*}{ Antinuclear antibodies } & $\begin{array}{l}\text { Mice with reduced gut bacteria developed nephritis more slowly and had } \\
\text { lower levels of circulating antinuclear antibodies (ANAs) compared to } \\
\text { the control group. }\end{array}$ & {$[67,69]$} \\
\hline & & $\begin{array}{l}\text { Germ-free lymphotoxin-deficient animals monocolonized with SFB } \\
\text { produced more ANAs than lymphotoxin-deficient controls } \\
\text { monocolonized with E. coli. }\end{array}$ & {$[68]$} \\
\hline \multirow{3}{*}{ CKD } & Endoxin & $\begin{array}{c}\text { Endotoxemia can lead to systemic inflammation, oxidative stress, cardiac } \\
\text { injury, and atherosclerosis. }\end{array}$ & {$[52]$} \\
\hline & $\begin{array}{l}\text { Uremic toxins (TMAO, IS, and } \\
\text { PCS) }\end{array}$ & $\begin{array}{c}\text { Uremic toxins cause inflammation and tubulointerstitial damage and } \\
\text { promote ROS production, tubulointerstitial damage, epithelial } \\
\text { cytotoxicity of proximal renal tubules, and progressive podocyte and } \\
\text { glomerular damage. }\end{array}$ & {$[51,96,97]$} \\
\hline & SCFAs & $\begin{array}{l}\text { Gut-derived SCFAs trigger hypertension through Olfr78 in the } \\
\text { peripheral blood vessels and renal afferent arterioles, which in turn leads } \\
\text { to renin secretion and regulation of peripheral resistance. }\end{array}$ & {$[23,84,101]$} \\
\hline \multirow{3}{*}{$\mathrm{DN}$} & Insulin resistance & $\begin{array}{l}\text { Intestinal dysbiosis is involved in insulin resistance and apoptosis of islet } \\
\text { cells in diabetes. }\end{array}$ & {$[7,10,11,116,118]$} \\
\hline & Activation of the RAS & $\begin{array}{c}\text { Ang II accelerates the progression of DN by inducing renal } \\
\text { vasoconstriction, promoting renal cell morphology, extracellular matrix } \\
\text { deposition, inflammatory cytokine secretion, and fibro-promoting } \\
\text { chemokines. }\end{array}$ & {$[125,126]$} \\
\hline & Uremic toxin & $\begin{array}{l}\text { Phenyl sulfate can cause proteinuria and podocyte injury in diabetic } \\
\text { mice. Inhibition of phenyl sulfate can reduce proteinuria in diabetic mice. }\end{array}$ & {$[122]$} \\
\hline IRI & $\begin{array}{l}\text { Bone marrow monocytes and } \\
\text { renal resident macrophages }\end{array}$ & $\begin{array}{l}\text { Applying antibiotics can diminish the gut microbiome and protect } \\
\text { against kidney IRI profoundly by reducing the maturation status of bone } \\
\text { marrow monocytes and } \mathrm{F} 4 / 80^{+} \text {renal resident macrophages. }\end{array}$ & {$[138]$} \\
\hline \multirow[t]{2}{*}{$\operatorname{IgAN}$} & TGF- $\beta$, BAFF, and APRIL & $\begin{array}{c}\text { Intestinal dysbiosis and chronic bacterial infections could stimulate } \\
\text { epithelial cells to produce BAFF and APRIL which could promote } \\
\text { excessive production of IgA. }\end{array}$ & {$[140,144-147]$} \\
\hline & Endoxin (LPS) & $\begin{array}{l}\text { LPS is involved in the presence of important features of IgAN } \\
\text { pathogenesis: hyperproduction and hypogalactosylation of IgA1. }\end{array}$ & {$[151]$} \\
\hline
\end{tabular}

immune complexes consisting of aberrantly glycosylated IgA1, IgG autoantibodies, and C3, which leads to glomerular inflammation [139]. The generation of IgA in the intestinal mucosa is a predominant immunological process that is critical for homeostasis between the intestinal microbiota and the local immunological environment $[140,141]$. Considering this, it could be hypothesized that gut dysbiosis and the abnormalities of the IgA mucosal immune system could be a significant element in the pathogenesis of IgAN [142].

The mucosal IgA is mainly produced in mesenteric lymph nodes (MLNs), Peyer's patches (PPs), and isolated lymphoid follicles (ILF) [140, 143]. The microenvironmental signals and controlling factors that drive the mass production of IgA in the intestine include the transforming growth factor- $\beta$ (TGF- $\beta$ ) [144], BAFF, and APRIL [140, 145-147], which can reveal the commensal dependence in the $\operatorname{IgA}$ switch and the IgA-driven pathology [148]. Intestinal dysbiosis and chronic bacterial infections could stimulate the epithelial cells to produce BAFF and APRIL that promote the excessive production of IgA. Furthermore, studies have reported the distinct differences of the gut microbiome and metabolome in IgAN patients and healthy controls [149, 150] (Figure 3).

Otherwise, the potential link between the gut microbiota and the pathogenesis of IgAN could be revealed in the inhibition of IgA1 glycosylation by bacterial LPS. Qin et al. suggest that LPS could significantly inhibit the chaperone Cosmc, which is essential for the activity of galactosyltransferase, via toll-like receptor 4 (TLR4) [151]. The low Cosmc mRNA expression restrains the galactosylation level of IgA1 in IgAN 
patients. Combined with the fact that the bacterial LPS itself can stimulate a local and systemic inflammatory response, LPS is involved in the presence of the important features of IgAN pathogenesis including hyperproduction and hypogalactosylation of IgA1 [151].

\section{Conclusion}

The gut microbiome can be considered a giant bioreactor of the human body, which holds a bidirectional relationship with the host. Human-produced factors, such as sIgA and AMPs, can affect and control the intestinal microbiota potentially. The colonization of intestinal microorganisms is a double-edged sword for the host, and it can elicit a variety of effects on the host's health and diseases. A healthy microbial community plays an indispensable role in supporting symbiotic homeostasis by helping the body in resisting sudden changes from the internal and external environment, metabolizing nutrients, and secreting hormones, promoting the maturation of immune cells, maintaining the integrity of the intestinal epithelial barrier, and preventing the colonization of pathogenic microorganisms. A great deal of basic research can also confirm the role of microbiota in the treatment of a variety of renal disorders. The morbid state of the kidney leads to gut microbial dysbiosis, and in turn, gut microbial alteration induces renal injury. Imbalanced microbial composition leads to intestinal barrier permeability increase, accumulation of uremic toxins, and impaired autoimmune tolerance. Microbial dysbiosis and increased intestinal barrier permeability would be involved in the translocation of pathogenic bacteria, bacterial endotoxins, and toxic metabolites. Circulating microbial components may not lead to a clinical manifestation of infection but instead promote many pathological changes. Bacterial endotoxins and toxic metabolites cause chronic inflammation by activating the NF- $\kappa \mathrm{B}$ pathway and promoting the production of proinflammatory chemokines; bacterial pathogens destroy autoimmune tolerance and induce autoimmunity by causing an imbalance in the Treg/Th17 ratio and abnormal activation of B cells. Moreover, the alternation of the gut microbiota can increase oxidative stress and induce hypertension (Table 1). These processes are thought to contribute to the further progression of kidney diseases. Clinical evidence that clarified the intricate pathogenesis of kidney diseases from a gut microbial perspective has opened the possibility for the development of innovative treatments in copious microbial pathways as both potential pharmacological targets and mediators for renal diseases. It may be helpful in the long term to modulate the composition of intestinal flora and restore the epithelial barriers through diet, probiotics, and antibiotics. Ranganathan et al. [152] found that BUN levels showed statistically apparent differences in outcomes $(P<0.05)$ between the placebo and probiotic treatment periods at all four sites (46 patients). Oral administration of Lactobacillus casei or L. acidophilus, both of which can be used as probiotics, reduced the production of phenolic and indole uremic toxins significantly in hemodialysis patients [153]. In another set of trials, CKD patients who took L. acidophilus orally had a significant decline in their levels of serum urea concentration (dimethylamine and nitrosodimethylamine) [154]. In addition, a randomized controlled clinical trial demonstrated that synbiotic therapy significantly alters uremic toxin, PCS, and a palpable shift in the stool microbiome (particularly with the increase of Bifidobacterium and the decrease of Ruminococcaceae) [155]. Parasitederived glycoprotein is involved in maintaining the balance of regulatory/effector $\mathrm{B}$ cells and desensitized renal effector function. Moreover, gut-derived SCFAs were proven to reduce inflammation, cellular oxidative stress, and immune cell infiltration and contribute to the regulation of DNA methylation status. These significant findings can contribute to the future development of treatment methods for renal diseases. Although numerous studies have been conducted, major advances are still needed to expand our understanding of the interaction mechanism between bacterial molecules and peripheral organs. Further investigations will be needed to prove the research achievement in animal models successfully in patients.

\section{Abbreviations}

SCFAs: Short-chain fatty acids

GPR41: $\quad$ G protein-coupled receptor 41

GPR43: $\quad$ G protein-coupled receptor 43

GPR109A: G protein-coupled receptor 109A

Treg cells: Regulatory T cells

NLRP3: $\quad$ NLR family pyrin domain containing 3

IL-18: Interleukin-18

HDACs: Histone deacetylases

IS: $\quad$ Indoxyl sulfate

PCS: $\quad$-Cresyl sulfate

TMAO: Trimethylamine $\mathrm{N}$-oxide

AhR: Aryl receptor

AMPs: Antimicrobial peptides

M cells: Microfold cells

DCs: $\quad$ Dendritic cells

Th1: $\quad$ Helper $\mathrm{T}$ cell 1

Th2: $\quad$ Helper $\mathrm{T}$ cell 2

Th17: $\quad$ Helper T cell 17

IL-4: Interleukin-4

IL-5: Interleukin-5

IL-6: $\quad$ Interleukin-6

IFN- $\gamma$ : Interferon- $\gamma$

APRIL: A proliferation-inducing ligand

BAFF: $\quad$ B cell-activating factor

sIgA: $\quad$ Secretory IgA

LN: Lupus nephritis

SLE: $\quad$ Systemic lupus erythematosus

TLRs: Toll-like receptors

TLR7: Toll-like receptor 7

TLR9: Toll-like receptor 9

IFN- $\alpha$ : $\quad$ Interferon- $\alpha$

APCs: Antigen-presenting cells

C3a: Complement component 3a

C5: $\quad$ Complement component 5

IL-17A: Interleukin-17A

IL-17F: Interleukin-17F

CXCL5: C-X-C motif chemokine 5 


$\begin{array}{ll}\text { CCL20: } & \text { Chemokine (C-C motif) ligand } 20 \\ \text { CCR6: } & \text { Chemokine receptor } 6 \\ \text { ROS: } & \text { Reactive oxygen species } \\ \text { CKD: } & \text { Chronic kidney disease } \\ \text { NF- } \kappa \text { B: } & \text { Nuclear factor-kappa B } \\ \text { LPSs: } & \text { Lipopolysaccharides } \\ \text { ESRD: } & \text { End-stage renal disease } \\ \text { Olfr78: } & \text { Olfactory receptor 78 } \\ \text { DN: } & \text { Diabetic nephropathy } \\ \text { ECM: } & \text { Extracellular matrix } \\ \text { FBG: } & \text { Fasting blood glucose } \\ \text { STZ: } & \text { Streptozotocin } \\ \text { RAS: } & \text { Renin-angiotensin system } \\ \text { Ang II: } & \text { Angiotensin II } \\ \text { IRI: } & \text { Ischemia-reperfusion injury } \\ \text { AKI: } & \text { Acute kidney injury } \\ \text { IgAN: } & \text { IgA nephropathy } \\ \text { MLNs: } & \text { Mesenteric lymph nodes } \\ \text { PPs: } & \text { Peyer's patches } \\ \text { ILF: } & \text { Isolated lymphoid follicles } \\ \text { TGF- } \beta: & \text { Transforming growth factor- } \beta \\ \text { TLR4: } & \text { Toll-like receptor } 4 .\end{array}$

\section{Ethical Approval}

This article does not contain any studies with human participants/animals performed by any of the authors.

\section{Conflicts of Interest}

All the authors declare that they have no conflict of interest.

\section{Authors' Contributions}

Mingxuan Chi, Kuai Ma, and Linjiang Song were involved in the conception of the study. Mingxuan Chi and Kuai Ma were involved in writing the article. Jing Wang, Zhaolun Ding, Yunlong Li, Shaomi Zhu, Xin Liang, Qinxiu Zhang, and Chi Liu critically revised the manuscript. All authors read and approved the final manuscript. Mingxuan Chi and Kuai Ma contributed equally to this work.

\section{Acknowledgments}

The work was supported by the Foundation of Popularization project Department of Sichuan Health commission (19PJYY0731), Foundation of "apricot grove scholar" of Chengdu University of Traditional Chinese Medicine (2019yky10), Xinlin scholars Science Foundation of Chengdu University of Traditional Chinese Medicine (ARQN2019007), and Science and technology project fund for Returned Students of Sichuan Province (00809504).

\section{References}

[1] A. Levin, M. Tonelli, J. Bonventre et al., "Global kidney health 2017 and beyond: a roadmap for closing gaps in care, research, and policy," Lancet, vol. 390, pp. 1888-1917, 2017.
[2] H. Abboud and W. L. Henrich, "Clinical practice. Stage IV chronic kidney disease," New England Journal of Medicine, vol. 362, no. 1, pp. 56-65, 2010.

[3] Q. Mu, H. Zhang, X. Liao et al., "Control of lupus nephritis by changes of gut microbiota," Microbiome, vol. 5, p. 73, 2017.

[4] S. Tao, L. Li, L. Li et al., "Understanding the gut-kidney axis among biopsy-proven diabetic nephropathy, type 2 diabetes mellitus and healthy controls: an analysis of the gut microbiota composition,” Acta Diabetologica, vol. 56, pp. 581-592, 2019.

[5] N. D. Vaziri, J. Wong, M. Pahl et al., "Chronic kidney disease alters intestinal microbial flora," Kidney International, vol. 83, pp. 308-315, 2013.

[6] J. Wong, Y. M. Piceno, T. Z. DeSantis, M. Pahl, G. L. Andersen, and N. D. Vaziri, "Expansion of urease- and uricase-containing, indole- and p-cresol-forming and contraction of shortchain fatty acid-producing intestinal microbiota in ESRD," American Journal of Nephrology, vol. 39, pp. 230-237, 2014.

[7] J. Henao-Mejia, E. Elinav, C. Jin et al., "Inflammasome-mediated dysbiosis regulates progression of NAFLD and obesity," Nature, vol. 482, pp. 179-185, 2012.

[8] W. H. Tang, T. Kitai, and S. L. Hazen, "Gut microbiota in cardiovascular health and disease," Circulation Research, vol. 120, pp. 1183-1196, 2017.

[9] C. A. Thaiss, S. Itav, D. Rothschild et al., "Persistent microbiome alterations modulate the rate of post-dieting weight regain," Nature, vol. 540, pp. 544-551, 2016.

[10] T. Vatanen, E. A. Franzosa, R. Schwager et al., "The human gut microbiome in early-onset type 1 diabetes from the TEDDY study," Nature, vol. 562, pp. 589-594, 2018.

[11] L. Zhao, F. Zhang, X. Ding et al., "Gut bacteria selectively promoted by dietary fibers alleviate type 2 diabetes," Science, vol. 359, pp. 1151-1156, 2018.

[12] R. A. Koeth, Z. Wang, B. S. Levison et al., "Intestinal microbiota metabolism of L-carnitine, a nutrient in red meat, promotes atherosclerosis," Nature Medicine, vol. 19, pp. 576-585, 2013.

[13] I. Rune, B. Rolin, C. Larsen et al., "Modulating the gut microbiota improves glucose tolerance, lipoprotein profile and atherosclerotic plaque development in ApoE-deficient mice," PLoS One, vol. 11, article e0146439, 2016.

[14] L. V. Hooper, T. Midtvedt, and J. I. Gordon, "How hostmicrobial interactions shape the nutrient environment of the mammalian intestine," Annual Review of Nutrition, vol. 22, pp. 283-307, 2002.

[15] S. H. Duncan, A. Belenguer, G. Holtrop, A. M. Johnstone, H. J. Flint, and G. E. Lobley, "Reduced dietary intake of carbohydrates by obese subjects results in decreased concentrations of butyrate and butyrate-producing bacteria in feces," Applied and Environmental Microbiology, vol. 73, no. 4, pp. 1073-1078, 2007.

[16] W. Wu, M. Sun, F. Chen et al., "Microbiota metabolite shortchain fatty acid acetate promotes intestinal IgA response to microbiota which is mediated by GPR43," Mucosal Immunology, vol. 10, pp. 946-956, 2017.

[17] Y. Furusawa, Y. Obata, S. Fukuda et al., "Commensal microbe-derived butyrate induces the differentiation of colonic regulatory T cells," Nature, vol. 504, no. 7480, pp. 446-450, 2013.

[18] L. Macia, J. Tan, A. T. Vieira et al., "Metabolite-sensing receptors GPR43 and GPR109A facilitate dietary fibre-induced gut homeostasis through regulation of the inflammasome," Nature Communications, vol. 6, p. 6734, 2015. 
[19] K. M. Maslowski, A. T. Vieira, A. Ng et al., "Regulation of inflammatory responses by gut microbiota and chemoattractant receptor GPR43," Nature, vol. 461, pp. 12821286, 2009.

[20] M. G. Rooks and W. S. Garrett, "Gut microbiota, metabolites and host immunity," Nature Reviews Immunology, vol. 16, pp. 341-352, 2016.

[21] V. Andrade-Oliveira, M. T. Amano, M. Correa-Costa et al., "Gut bacteria products prevent AKI induced by ischemiareperfusion," Journal of the American Society of Nephrology, vol. 26, no. 8, pp. 1877-1888, 2015.

[22] R. J. Felizardo, A. Castoldi, V. Andrade-Oliveira, and N. O. S. Câmara, "The microbiota and chronic kidney diseases: a double-edged sword," Clinical \& Translational Immunology, vol. 5, no. 6, article e86, 2016.

[23] J. L. Pluznick, R. J. Protzko, H. Gevorgyan et al., "Olfactory receptor responding to gut microbiota-derived signals plays a role in renin secretion and blood pressure regulation," Proceedings of the National Academy of Sciences of the United States of America, vol. 110, pp. 4410-4415, 2013.

[24] J. Yang, H. Dong, Y. Wang et al., "Cordyceps cicadae polysaccharides ameliorated renal interstitial fibrosis in diabetic nephropathy rats by repressing inflammation and modulating gut microbiota dysbiosis," International Journal of Biological Macromolecules, vol. 163, pp. 442-456, 2020.

[25] M. B. Geuking, J. Cahenzli, M. A. Lawson et al., "Intestinal bacterial colonization induces mutualistic regulatory $\mathrm{T}$ cell responses," Immunity, vol. 34, no. 5, pp. 794-806, 2011.

[26] S. Hapfelmeier, M. A. Lawson, E. Slack et al., "Reversible microbial colonization of germ-free mice reveals the dynamics of IgA immune responses," Science, vol. 328, no. 5986, pp. 1705-1709, 2010.

[27] S. Moghadamrad, K. D. McCoy, M. B. Geuking et al., "Attenuated portal hypertension in germ-free mice: function of bacterial flora on the development of mesenteric lymphatic and blood vessels," Hepatology, vol. 61, pp. 1685-1695, 2015.

[28] A. Sabatino, G. Regolisti, I. Brusasco, A. Cabassi, S. Morabito, and E. Fiaccadori, "Alterations of intestinal barrier and microbiota in chronic kidney disease," Nephrology, Dialysis, Transplantation, vol. 30, pp. 924-933, 2015.

[29] A. Adak and M. R. Khan, "An insight into gut microbiota and its functionalities," Cellular and Molecular Life Sciences, vol. 76, no. 3, pp. 473-493, 2019.

[30] M. M. France and J. R. Turner, "The mucosal barrier at a glance," Journal of Cell Science, vol. 130, no. 2, pp. 307-314, 2017.

[31] R. E. Salvo, C. C. Alonso, C. C. Pardo, B. M. Casado, and M. Vicario, "The intestinal barrier function and its involvement in digestive disease," Revista Española de Enfermedades Digestivas, vol. 107, pp. 686-696, 2015.

[32] T. Tanoue, S. Morita, D. R. Plichta et al., "A defined commensal consortium elicits CD8 T cells and anti-cancer immunity," Nature, vol. 565, pp. 600-605, 2019.

[33] B. Meijers, R. Farre, S. Dejongh, M. Vicario, and P. Evenepoel, "Intestinal barrier function in chronic kidney disease," Toxins, vol. 10, no. 7, p. 298, 2018.

[34] T. Pelaseyed, J. H. Bergstrom, J. K. Gustafsson et al., “The mucus and mucins of the goblet cells and enterocytes provide the first defense line of the gastrointestinal tract and interact with the immune system," Immunological Reviews, vol. 260, pp. 8-20, 2014.
[35] M. Cella, A. Fuchs, W. Vermi et al., "A human natural killer cell subset provides an innate source of IL-22 for mucosal immunity," Nature, vol. 457 , no. 7230 , pp. $722-$ $725,2009$.

[36] A. S. Ismail, C. L. Behrendt, and L. V. Hooper, "Reciprocal interactions between commensal bacteria and gamma delta intraepithelial lymphocytes during mucosal injury," Journal of Immunology, vol. 182, pp. 3047-3054, 2009.

[37] M. Rescigno, M. Urbano, B. Valzasina et al., "Dendritic cells express tight junction proteins and penetrate gut epithelial monolayers to sample bacteria," Nature Immunology, vol. 2, pp. 361-367, 2001.

[38] Y. Wang, E. P. Koroleva, A. A. Kruglov et al., "Lymphotoxin beta receptor signaling in intestinal epithelial cells orchestrates innate immune responses against mucosal bacterial infection," Immunity, vol. 32, pp. 403-413, 2010.

[39] O. S. Sakhon, B. Ross, V. Gusti, A. J. Pham, K. Vu, and D. D. Lo, " $\mathrm{M}$ cell-derived vesicles suggest a unique pathway for trans-epithelial antigen delivery," Tissue Barriers, vol. 3, article e1004975, 2015.

[40] I. I. Ivanov, R. L. Frutos, N. Manel et al., "Specific microbiota direct the differentiation of IL-17-producing T-helper cells in the mucosa of the small intestine," Cell Host \& Microbe, vol. 4, pp. 337-349, 2008.

[41] S. K. Mazmanian, C. H. Liu, A. O. Tzianabos, and D. L. Kasper, "An immunomodulatory molecule of symbiotic bacteria directs maturation of the host immune system," Cell, vol. 122, pp. 107-118, 2005.

[42] N. Rother and J. van der Vlag, "Disturbed T cell signaling and altered Th17 and regulatory T cell subsets in the pathogenesis of systemic lupus erythematosus," Frontiers in Immunology, vol. 6, p. 610, 2015.

[43] M. Levy, C. A. Thaiss, D. Zeevi et al., "Microbiota-modulated metabolites shape the intestinal microenvironment by regulating NLRP6 inflammasome signaling," Cell, vol. 163, pp. 1428-1443, 2015.

[44] L. A. David, C. F. Maurice, R. N. Carmody et al., "Diet rapidly and reproducibly alters the human gut microbiome," Nature, vol. 505, no. 7484, pp. 559-563, 2014.

[45] J. J. Faith, J. L. Guruge, M. Charbonneau et al., "The longterm stability of the human gut microbiota," Science, vol. 341, no. 6141, 2013.

[46] A. Nallu, S. Sharma, A. Ramezani, J. Muralidharan, and D. Raj, "Gut microbiome in chronic kidney disease: challenges and opportunities," Translational Research, vol. 179, pp. 24-37, 2017.

[47] A. P. Hendrickx, J. Top, J. R. Bayjanov et al., "Antibioticdriven dysbiosis mediates intraluminal agglutination and alternative segregation of Enterococcus faecium from the intestinal epithelium," MBio, vol. 6, no. 6, p. e01346, 2015.

[48] H. Tanaka, M. Takechi, H. Kiyonari, G. Shioi, A. Tamura, and S. Tsukita, "Intestinal deletion of claudin-7 enhances paracellular organic solute flux and initiates colonic inflammation in mice," Gut, vol. 64, pp. 1529-1538, 2015.

[49] C. A. Thaiss, M. Levy, I. Grosheva et al., "Hyperglycemia drives intestinal barrier dysfunction and risk for enteric infection," Science, vol. 359, pp. 1376-1383, 2018.

[50] S. Zeissig, N. Burgel, D. Gunzel et al., "Changes in expression and distribution of claudin 2, 5 and 8 lead to discontinuous tight junctions and barrier dysfunction in active Crohn's disease," Gut, vol. 56, pp. 61-72, 2007. 
[51] C. J. Lin, V. Wu, P. C. Wu, and C. J. Wu, "Meta-analysis of the associations of p-cresyl sulfate (PCS) and indoxyl sulfate (IS) with cardiovascular events and all-cause mortality in patients with chronic renal failure," PLoS One, vol. 10, article e0132589, 2015.

[52] C. W. McIntyre, L. E. Harrison, M. T. Eldehni et al., "Circulating endotoxemia: a novel factor in systemic inflammation and cardiovascular disease in chronic kidney disease," Clinical Journal of the American Society of Nephrology, vol. 6, pp. 133-141, 2011.

[53] V. M. Shaheen, M. Satoh, H. B. Richards et al., "Immunopathogenesis of environmentally induced lupus in mice," Environmental Health Perspectives, vol. 107, Suppl 5, pp. 723-727, 1999.

[54] S. V. Parikh, S. Almaani, S. Brodsky, and B. H. Rovin, "Update on lupus nephritis: core curriculum 2020," American Journal of Kidney Diseases, vol. 76, pp. 265-281, 2020.

[55] T. Koutsokeras and T. Healy, "Systemic lupus erythematosus and lupus nephritis," Nature Reviews Drug Discovery, vol. 13, pp. 173-174, 2014.

[56] Z. He, T. Shao, H. Li, Z. Xie, and C. Wen, "Alterations of the gut microbiome in Chinese patients with systemic lupus erythematosus," Gut pathogens, vol. 8, no. 1, p. 64, 2016.

[57] A. Hevia, C. Milani, P. Lopez et al., "Intestinal dysbiosis associated with systemic lupus erythematosus," MBio, vol. 5, no. 5, p. e01548, 2014.

[58] X. M. Luo, M. R. Edwards, Q. Mu et al., "Gut microbiota in human systemic lupus erythematosus and a mouse model of lupus," Applied and Environmental Microbiology, vol. 84, 2018.

[59] T. A. van der Meulen, H. J. M. Harmsen, A. V. Vila et al., "Shared gut, but distinct oral microbiota composition in primary Sjogren's syndrome and systemic lupus erythematosus," Journal of Autoimmunity, vol. 97, pp. 77-87, 2019.

[60] F. Wei, H. Xu, C. Yan, C. Rong, B. Liu, and H. Zhou, "Changes of intestinal flora in patients with systemic lupus erythematosus in northeast China," PLoS One, vol. 14, article e0213063, 2019.

[61] H. Zhang, X. Liao, J. B. Sparks, and X. M. Luo, "Dynamics of gut microbiota in autoimmune lupus," Applied and Environmental Microbiology, vol. 80, pp. 7551-7560, 2014.

[62] H. Neuman and O. Koren, "The gut microbiota: a possible factor influencing systemic lupus erythematosus," Current Opinion in Rheumatology, vol. 29, pp. 374-377, 2017.

[63] P. Lopez, B. Sanchez, A. Margolles, and A. Suarez, "Intestinal dysbiosis in systemic lupus erythematosus: cause or consequence?" Current Opinion in Rheumatology, vol. 28, pp. 515-522, 2016.

[64] F. Capolunghi, M. M. Rosado, S. Cascioli et al., "Pharmacological inhibition of TLR9 activation blocks autoantibody production in human B cells from SLE patients," Rheumatology, vol. 49, no. 12, pp. 2281-2289, 2010.

[65] B. D. Lyn-Cook, C. Xie, J. Oates et al., "Increased expression of toll-like receptors (TLRs) 7 and 9 and other cytokines in systemic lupus erythematosus (SLE) patients: ethnic differences and potential new targets for therapeutic drugs," Molecular Immunology, vol. 61, pp. 38-43, 2014.

[66] A. Kimura and T. Kishimoto, "IL-6: regulator of Treg/Th17 balance," European Journal of Immunology, vol. 40, pp. 1830-1835, 2010.

[67] B. M. Johnson, M. C. Gaudreau, M. M. Al-Gadban, R. Gudi, and C. Vasu, "Impact of dietary deviation on disease progres- sion and gut microbiome composition in lupus-prone SNF1 mice," Clinical and Experimental Immunology, vol. 181, pp. 323-337, 2015.

[68] T. Van de Wiele, J. T. Van Praet, M. Marzorati, M. B. Drennan, and D. Elewaut, "How the microbiota shapes rheumatic diseases," Nature Reviews Rheumatology, vol. 12, pp. 398411, 2016.

[69] J. T. Van Praet, E. Donovan, I. Vanassche et al., "Commensal microbiota influence systemic autoimmune responses," The EMBO Journal, vol. 34, pp. 466-474, 2015.

[70] O. Avni and O. Koren, "Molecular (Me)micry?," Cell Host \& Microbe, vol. 23, no. 5, pp. 576-578, 2018.

[71] T. M. Greiling, C. Dehner, X. Chen et al., "Commensal orthologs of the human autoantigen Ro60 as triggers of autoimmunity in lupus," Science Translational Medicine, vol. 10, no. 434, p. eaan2306, 2018.

[72] D. Azzouz, A. Omarbekova, A. Heguy et al., "Lupus nephritis is linked to disease-activity associated expansions and immunity to a gut commensal," Annals of the Rheumatic Diseases, vol. 78, no. 7, pp. 947-956, 2019.

[73] S. K. Kwok and G. C. Tsokos, "New insights into the role of renal resident cells in the pathogenesis of lupus nephritis," The Korean Journal of Internal Medicine, vol. 33, pp. 284289, 2018.

[74] S. V. Parikh and B. H. Rovin, "Current and emerging therapies for lupus nephritis," Journal of the American Society of Nephrology, vol. 27, no. 10, pp. 2929-2939, 2016.

[75] G. L. V. de Oliveira, A. Z. Leite, B. S. Higuchi, M. I. Gonzaga, and V. S. Mariano, "Intestinal dysbiosis and probiotic applications in autoimmune diseases," Immunology, vol. 152, no. 1, pp. 1-12, 2017.

[76] S. A. Esmaeili, M. Mahmoudi, A. A. Momtazi, A. Sahebkar, H. Doulabi, and M. Rastin, "Tolerogenic probiotics: potential immunoregulators in systemic lupus erythematosus," Journal of Cellular Physiology, vol. 232, no. 8, pp. 1994-2007, 2017.

[77] D. T. Rodgers, M. A. McGrath, M. A. Pineda et al., "The parasitic worm product ES-62 targets myeloid differentiation factor 88-dependent effector mechanisms to suppress antinuclear antibody production and proteinuria in $\mathrm{MRL} / \mathrm{lpr}$ mice," Arthritis \& Rhematology, vol. 67, pp. 1023-1035, 2015.

[78] K. U. Eckardt, J. Coresh, O. Devuyst et al., "Evolving importance of kidney disease: from subspecialty to global health burden," The Lancet, vol. 382, no. 9887, pp. 158-169, 2013.

[79] H. J. Anders, K. Andersen, and B. Stecher, "The intestinal microbiota, a leaky gut, and abnormal immunity in kidney disease," Kidney International, vol. 83, no. 6, pp. 10101016, 2013.

[80] A. Ramezani and D. S. Raj, "The gut microbiome, kidney disease, and targeted interventions," Journal of the American Society of Nephrology, vol. 25, no. 4, pp. 657-670, 2014.

[81] T. Peschel, M. Schonauer, H. Thiele, S. D. Anker, G. Schuler, and J. Niebauer, "Invasive assessment of bacterial endotoxin and inflammatory cytokines in patients with acute heart failure," European Journal of Heart Failure, vol. 5, pp. 609-614, 2003.

[82] N. D. Vaziri, Y. Y. Zhao, and M. V. Pahl, "Altered intestinal microbial flora and impaired epithelial barrier structure and function in CKD: the nature, mechanisms, consequences and potential treatment," Nephrology, Dialysis, Transplantation, vol. 31, pp. 737-746, 2016. 
[83] R. L. Converse Jr., T. N. Jacobsen, R. D. Toto et al., "Sympathetic overactivity in patients with chronic renal failure," New England Journal of Medicine, vol. 327, no. 27, pp. 1912-1918, 1992.

[84] M. M. Santisteban, Y. Qi, J. Zubcevic et al., "Hypertensionlinked pathophysiological alterations in the gut," Circulation Research, vol. 120, pp. 312-323, 2017.

[85] M. Ardalan and S. Z. Vahed, "Gut microbiota and renal transplant outcome," Biomedicine \& Pharmacotherapy, vol. 90, pp. 229-236, 2017.

[86] K. Andersen, M. S. Kesper, J. A. Marschner et al., "Intestinal dysbiosis, barrier dysfunction, and bacterial translocation account for CKD-related systemic inflammation," Journal of the American Society of Nephrology, vol. 28, no. 1, pp. 7683, 2017.

[87] X. Lai, A. H. Zhang, S. Y. Chen et al., "Outcomes of stage 1-5 chronic kidney disease in mainland China," Renal Failure, vol. 36, pp. 520-525, 2014.

[88] R. Saran, B. Robinson, K. C. Abbott et al., "US renal data system 2017 annual data report: epidemiology of kidney disease in the United States," American Journal of Kidney Diseases, vol. 71, no. A7, p. 2018, 2017.

[89] S. K. Biswas and E. Lopez-Collazo, "Endotoxin tolerance: new mechanisms, molecules and clinical significance," Trends in Immunology, vol. 30, no. 10, pp. 475-487, 2009.

[90] P. A. Aronov, F. J. Luo, N. S. Plummer et al., "Colonic contribution to uremic solutes," Journal of the American Society of Nephrology, vol. 22, no. 9, pp. 1769-1776, 2011.

[91] C. Y. Sun, S. C. Chang, and M. S. Wu, "Uremic toxins induce kidney fibrosis by activating intrarenal renin-angiotensinaldosterone system associated epithelial-to-mesenchymal transition," PLoS One, vol. 7, article e34026, 2012.

[92] Y. Huang, S. Wongamorntham, J. Kasting et al., "Renin increases mesangial cell transforming growth factor-betal and matrix proteins through receptor-mediated, angiotensin II-independent mechanisms," Kidney International, vol. 69, pp. 105-113, 2006.

[93] E. Schepers, N. Meert, G. Glorieux, J. Goeman, J. Van der Eycken, and R. Vanholder, "P-Cresylsulphate, the main in vivo metabolite of $\mathrm{p}$-cresol, activates leucocyte free radical production," Nephrology, Dialysis, Transplantation, vol. 22, pp. 592-596, 2007.

[94] C. Y. Sun, S. C. Chang, and M. S. Wu, "Suppression of Klotho expression by protein-bound uremic toxins is associated with increased DNA methyltransferase expression and DNA hypermethylation," Kidney International, vol. 81, pp. 640-650, 2012.

[95] S. Klammt, H. J. Wojak, A. Mitzner et al., “Albumin-binding capacity $(\mathrm{ABiC})$ is reduced in patients with chronic kidney disease along with an accumulation of protein-bound uraemic toxins," Nephrology, Dialysis, Transplantation, vol. 27, pp. 2377-2383, 2012.

[96] A. Ramezani, Z. A. Massy, B. Meijers, P. Evenepoel, R. Vanholder, and D. S. Raj, "Role of the gut microbiome in uremia: a potential therapeutic target," American Journal of Kidney Diseases, vol. 67, pp. 483-498, 2016.

[97] O. Ichii, S. Otsuka-Kanazawa, T. Nakamura et al., "Podocyte injury caused by indoxyl sulfate, a uremic toxin and arylhydrocarbon receptor ligand," PLoS One, vol. 9, article e108448, 2014.

[98] M. V. Rao, Y. Qiu, C. Wang, and G. Bakris, "Hypertension and CKD: Kidney Early Evaluation Program (KEEP) and
National Health and Nutrition Examination Survey (NHANES), 1999-2004," American Journal of Kidney Diseases, vol. 51, pp. S30-S37, 2008.

[99] D. J. Durgan, B. P. Ganesh, J. L. Cope et al., "Role of the gut microbiome in obstructive sleep apnea-induced hypertension," Hypertension, vol. 67, no. 2, pp. 469-474, 2016.

[100] J. Li, F. Zhao, Y. Wang et al., "Gut microbiota dysbiosis contributes to the development of hypertension," Microbiome, vol. 5, p. 14, 2017.

[101] B. Mell, V. R. Jala, A. V. Mathew et al., "Evidence for a link between gut microbiota and hypertension in the Dahl rat," Physiological Genomics, vol. 47, pp. 187-197, 2015.

[102] Y. Qi, J. M. Aranda, V. Rodriguez, M. K. Raizada, and C. J. Pepine, "Impact of antibiotics on arterial blood pressure in a patient with resistant hypertension - a case report," International Journal of Cardiology, vol. 201, pp. 157-158, 2015.

[103] T. Yang, M. M. Santisteban, V. Rodriguez et al., "Gut dysbiosis is linked to hypertension," Hypertension, vol. 65, pp. 1331-1340, 2015.

[104] N. Natarajan, D. Hori, S. Flavahan et al., "Microbial short chain fatty acid metabolites lower blood pressure via endothelial G protein-coupled receptor 41," Physiological Genomics, vol. 48, pp. 826-834, 2016.

[105] J. Pluznick, "A novel SCFA receptor, the microbiota, and blood pressure regulation," Gut Microbes, vol. 5, pp. 202207, 2014.

[106] S. Hamdi, G. M. Rousseau, S. J. Labrie et al., "Characterization of two polyvalent phages infecting _Enterobacteriaceae_," Scientific Reports, vol. 7, no. 1, p. 40349, 2017.

[107] Y. Xu, Y. Liu, Y. Liu, J. Pei, S. Yao, and C. Cheng, "Bacteriophage therapy against Enterobacteriaceae," Virologica Sinica, vol. 30, pp. 11-18, 2015.

[108] M. Hida, Y. Aiba, S. Sawamura, N. Suzuki, T. Satoh, and Y. Koga, "Inhibition of the accumulation of uremic toxins in the blood and their precursors in the feces after oral administration of Lebenin, a lactic acid bacteria preparation, to uremic patients undergoing hemodialysis," Nephron, vol. 74, no. 2, pp. 349-355, 1996.

[109] L. Lobel, Y. G. Cao, K. Fenn, J. N. Glickman, and W. S. Garrett, "Diet posttranslationally modifies the mouse gut microbial proteome to modulate renal function," Science, vol. 369, pp. 1518-1524, 2020.

[110] A. P. Lakshmanan, Z.'a. M. Al, B. H. Ali, and A. Terranegra, "The influence of the prebiotic gum acacia on the intestinal microbiome composition in rats with experimental chronic kidney disease," Biomedicine \& Pharmacotherapy, vol. 133, p. 110992, 2021.

[111] P. Saeedi, I. Petersohn, P. Salpea et al., "Global and regional diabetes prevalence estimates for 2019 and projections for 2030 and 2045: results from the International Diabetes Federation Diabetes Atlas," Diabetes Research and Clinical Practice, vol. 157, p. 107843, 2019.

[112] American Diabetes Association, "Diagnosis and classification of diabetes mellitus," Diabetes Care, vol. 37, Supplement 1, pp. S81-S90, 2014.

[113] K. Umanath and J. B. Lewis, "Update on diabetic nephropathy: core curriculum 2018," American Journal of Kidney Diseases, vol. 71, pp. 884-895, 2018.

[114] O. Aouacheri, S. Saka, M. Krim, A. Messaadia, and I. Maidi, "The investigation of the oxidative stress-related parameters 
in type 2 diabetes mellitus," Canadian Journal of Diabetes, vol. 39, no. 1, pp. 44-49, 2015.

[115] N. Esser, S. Legrand-Poels, J. Piette, A. J. Scheen, and N. Paquot, "Inflammation as a link between obesity, metabolic syndrome and type 2 diabetes," Diabetes Research and Clinical Practice, vol. 105, no. 2, pp. 141-150, 2014.

[116] J. Y. Yang, Y. S. Lee, Y. Kim et al., "Gut commensal Bacteroides acidifaciens prevents obesity and improves insulin sensitivity in mice," Mucosal Immunology, vol. 10, pp. 104-116, 2017.

[117] G. P. Donaldson, S. M. Lee, and S. K. Mazmanian, "Gut biogeography of the bacterial microbiota," Nature Reviews Microbiology, vol. 14, no. 1, pp. 20-32, 2016.

[118] C. He, Y. Shan, and W. Song, "Targeting gut microbiota as a possible therapy for diabetes," Nutrition Research, vol. 35, no. 5, pp. 361-367, 2015.

[119] J. Zhao, Q.-L. Zhang, J.-H. Shen, K. Wang, and J. Liu, "Magnesium lithospermate $\mathrm{B}$ improves the gut microbiome and bile acid metabolic profiles in a mouse model of diabetic nephropathy," Acta Pharmacologica Sinica, vol. 40, pp. 507513, 2018.

[120] M. A. Croxen, R. J. Law, R. Scholz, K. M. Keeney, M. Wlodarska, and B. B. Finlay, "Recent advances in understanding enteric pathogenic Escherichia coli," Clinical Microbiology Reviews, vol. 26, no. 4, pp. 822-880, 2013.

[121] S. de Kort, D. Keszthelyi, and A. A. Masclee, "Leaky gut and diabetes mellitus: what is the link?," Obesity Reviews, vol. 12, no. 6, pp. 449-458, 2011.

[122] K. Kikuchi, D. Saigusa, Y. Kanemitsu et al., "Gut microbiome-derived phenyl sulfate contributes to albuminuria in diabetic kidney disease," Nature Communications, vol. 10, p. 1835, 2019.

[123] J. Donate-Correa, E. Martin-Nunez, M. Muros-de-Fuentes, C. Mora-Fernandez, and J. F. Navarro-Gonzalez, "Inflammatory cytokines in diabetic nephropathy," Journal of Diabetes Research, vol. 2015, Article ID 948417, 9 pages, 2015.

[124] C. C. Lu, Z. B. Hu, R. Wang et al., "Gut microbiota dysbiosisinduced activation of the intrarenal renin-angiotensin system is involved in kidney injuries in rat diabetic nephropathy," Acta Pharmacologica Sinica, vol. 41, pp. 1111-1118, 2020.

[125] J. Tamura, A. Konno, Y. Hashimoto, and Y. Kon, "Upregulation of renal renin-angiotensin system in mouse diabetic nephropathy," The Japanese Journal of Veterinary Research, vol. 53, pp. 13-26, 2005.

[126] Z. B. Hu, J. Lu, P. P. Chen et al., "Dysbiosis of intestinal microbiota mediates tubulointerstitial injury in diabetic nephropathy via the disruption of cholesterol homeostasis," Theranostics, vol. 10, pp. 2803-2816, 2020.

[127] G. L. Bakris, R. Agarwal, J. C. Chan et al., "Effect of finerenone on albuminuria in patients with diabetic nephropathy," JAMA, vol. 314, no. 9, pp. 884-894, 2015.

[128] P. Khajehdehi, M. Pakfetrat, K. Javidnia et al., "Oral supplementation of turmeric attenuates proteinuria, transforming growth factor-beta and interleukin-8 levels in patients with overt type 2 diabetic nephropathy: a randomized, doubleblind and placebo-controlled study," Scandinavian Journal of Urology and Nephrology, vol. 45, pp. 365-370, 2011.

[129] J. M. Lachin, G. Viberti, B. Zinman et al., "Renal function in type 2 diabetes with rosiglitazone, metformin, and glyburide monotherapy," Clinical Journal of the American Society of Nephrology, vol. 6, pp. 1032-1040, 2011.
[130] H. Panwar, H. M. Rashmi, V. K. Batish, and S. Grover, "Probiotics as potential biotherapeutics in the management of type 2 diabetes - prospects and perspectives," Diabetes/Metabolism Research and Reviews, vol. 29, pp. 103-112, 2013.

[131] Y. Feng, H. Weng, L. Ling et al., "Modulating the gut microbiota and inflammation is involved in the effect of _Bupleurum_ polysaccharides against diabetic nephropathy in mice," International Journal of Biological Macromolecules, vol. 132, pp. 1001-1011, 2019.

[132] H. J. Zheng, J. Guo, Q. Jia et al., "The effect of probiotic and synbiotic supplementation on biomarkers of inflammation and oxidative stress in diabetic patients: a systematic review and meta-analysis of randomized controlled trials," Pharmacological Research, vol. 142, pp. 303-313, 2019.

[133] H. Wei, L. Wang, Z. An et al., "QiDiTangShen granules modulated the gut microbiome composition and improved bile acid pro fi les in a mouse model of diabetic nephropathy," Biomedicine \& Pharmacotherapy, vol. 133, p. 111061, 2021.

[134] H. Fujii, M. Iwase, T. Ohkuma et al., "Impact of dietary fiber intake on glycemic control, cardiovascular risk factors and chronic kidney disease in Japanese patients with type 2 diabetes mellitus: the Fukuoka Diabetes Registry," Nutrition Journal, vol. 12, no. 1, p. 159, 2013.

[135] N. Chatauret, L. Badet, B. Barrou, and T. Hauet, "Ischemiereperfusion : de la biologie cellulaire a la lesion renale," Progrès en Urologie, vol. 24, Supplement 1, pp. S4-S12, 2014.

[136] R. Thadhani, M. Pascual, and J. V. Bonventre, "Acute renal failure," The New England Journal of Medicine, vol. 334, pp. 1448-1460, 1996.

[137] H. R. Jang, G. J. Ko, B. A. Wasowska, and H. Rabb, “The interaction between ischemia-reperfusion and immune responses in the kidney," Journal of Molecular Medicine (Berlin, Germany), vol. 87, pp. 859-864, 2009.

[138] D. Emal, E. Rampanelli, I. Stroo et al., "Depletion of gut microbiota protects against renal ischemia-reperfusion injury," Journal of the American Society of Nephrology, vol. 28, no. 5, pp. 1450-1461, 2017.

[139] J. C. Rodrigues, M. Haas, and H. N. Reich, "IgA nephropathy," Clinical Journal of the American Society of Nephrology, vol. 12, pp. 677-686, 2017.

[140] S. Fagarasan, S. Kawamoto, O. Kanagawa, and K. Suzuki, "Adaptive immune regulation in the gut: T cell-dependent and T cell-independent IgA synthesis," Annual Review of Immunology, vol. 28, no. 1, pp. 243-273, 2010.

[141] A. J. Macpherson, K. D. McCoy, F. E. Johansen, and P. Brandtzaeg, "The immune geography of IgA induction and function," Mucosal Immunology, vol. 1, pp. 11-22, 2008.

[142] J. Floege and J. Feehally, "The mucosa-kidney axis in IgA nephropathy," Nature Reviews Nephrology, vol. 12, no. 3, pp. 147-156, 2016.

[143] P. Brandtzaeg, "Function of mucosa-associated lymphoid tissue in antibody formation," Immunological Investigations, vol. 39, no. 4-5, pp. 303-355, 2010.

[144] T. Iwasato, H. Arakawa, A. Shimizu, T. Honjo, and H. Yamagishi, "Biased distribution of recombination sites within $S$ regions upon immunoglobulin class switch recombination induced by transforming growth factor beta and lipopolysaccharide," The Journal of Experimental Medicine, vol. 175, pp. 1539-1546, 1992.

[145] E. Castigli, S. Scott, F. Dedeoglu et al., "Impaired IgA class switching in APRIL-deficient mice," Proceedings of the 
National Academy of Sciences, vol. 101, no. 11, pp. 39033908, 2004

[146] E. Castigli, S. A. Wilson, S. Scott et al., "TACI and BAFF-R mediate isotype switching in B cells," Journal of Experimental Medicine, vol. 201, no. 1, pp. 35-39, 2005.

[147] M. B. Litinskiy, B. Nardelli, D. M. Hilbert et al., "DCs induce CD40-independent immunoglobulin class switching through BLyS and APRIL," Nature Immunology, vol. 3, pp. 822-829, 2002.

[148] D. D. McCarthy, J. Kujawa, C. Wilson et al., "Mice overexpressing BAFF develop a commensal flora-dependent, IgAassociated nephropathy," The Journal of Clinical Investigation, vol. 121, pp. 3991-4002, 2011.

[149] M. De Angelis, E. Montemurno, M. Piccolo et al., "Microbiota and metabolome associated with immunoglobulin A nephropathy (IgAN)," PLoS One, vol. 9, no. 6, article e99006, 2014.

[150] M. Piccolo, M. De Angelis, G. Lauriero et al., "Salivary microbiota associated with immunoglobulin A nephropathy," Microbial Ecology, vol. 70, pp. 557-565, 2015.

[151] W. Qin, X. Zhong, J. M. Fan, Y. J. Zhang, X. R. Liu, and X. Y. $\mathrm{Ma}$, "External suppression causes the low expression of the Cosmc gene in IgA nephropathy," Nephrology, Dialysis, Transplantation, vol. 23, pp. 1608-1614, 2008.

[152] N. Ranganathan, P. Ranganathan, E. A. Friedman et al., "Pilot study of probiotic dietary supplementation for promoting healthy kidney function in patients with chronic kidney disease," Advances in Therapy, vol. 27, pp. 634-647, 2010.

[153] M. Pavan, "Influence of prebiotic and probiotic supplementation on the progression of chronic kidney disease," Minerva Urologica e Nefrologica, vol. 68, pp. 222-226, 2016.

[154] P. V. Miranda Alatriste, A. R. Urbina, C. O. Gomez Espinosa, and L. Espinosa Cuevas Mde, "Effect of probiotics on human blood urea levels in patients with chronic renal failure," Nutrición Hospitalaria, vol. 29, pp. 582-590, 2014.

[155] M. Rossi, D. W. Johnson, M. Morrison et al., "Synbiotics Easing Renal Failure by Improving Gut Microbiology (SYNERGY): a randomized trial," Clinical Journal of the American Society of Nephrology, vol. 11, pp. 223-231, 2016. 